\title{
Stirling Numbers and Spin-Euler Polynomials
}

\author{
D. Eelbode
}

\section{CONTENTS}

1. Introduction

2. Spin-Euler Polynomials of Types $(k, 0)$ and $(0, k)$

3. Commutation Behavior of Spin-Euler Polynomials

4. Spin-Euler Polynomials of type $(k, k)$

5. Spin-Euler Polynomials of Type $(k, l) \in \mathbb{N}_{0}^{2}(k \neq l)$

Acknowledgments

References
2000 AMS Subject Classification: Primary 30G35;

Secondary 32W50, 15A66

Keywords: Fischer decomposition, Hermitian Clifford analysis, Stirling numbers
The Fischer decomposition on $\mathbb{R}^{n}$ gives the decomposition of arbitrary homogeneous polynomials in $n$ variables $\left(x_{1}, \ldots, x_{n}\right)$ in terms of harmonic homogeneous polynomials. In classical Clifford analysis a refinement was obtained, giving a decomposition in terms of monogenic polynomials, i.e., homogeneous null solutions for the Dirac operator (a vector-valued differential operator factorizing the Laplacian $\Delta_{n}$ on $\mathbb{R}^{n}$ ). In this paper the building blocks for the Fischer decomposition in the Hermitian Clifford setting are determined, yielding a new refinement of harmonic analysis on $\mathbb{R}^{2 n}$ involving complex Dirac operators commuting with the action of the unitary group.

\section{INTRODUCTION}

Clifford analysis is a function theory that is often considered as a generalization to higher dimensions of the theory of complex holomorphic functions $f(z)$ in the plane. The analogue of the Cauchy-Riemann operator $\partial_{z}$ is the Dirac operator $\underline{\partial}$, a vector-valued first-order differential operator factorizing the Laplacian $\Delta_{n}$ on $\mathbb{R}^{n}$, and generalized holomorphic functions are called monogenic functions. These are Clifford-algebra-valued functions $f(\underline{x})$ defined on open regions $\Omega \subset \mathbb{R}^{n}$ satisfying $\underline{\partial} f=0$. This Clifford algebra is then defined as the $2^{n}$ dimensional algebra obtained by endowing the orthogonal space $\left(\mathbb{R}^{n}, Q_{n}\right)$, where $Q_{n}(\underline{x})=|\underline{x}|^{2}=\sum_{i=1}^{n} x_{i}^{2}$ for all $\underline{x} \in \mathbb{R}^{n}$, with the following multiplication rules: $e_{i} e_{j}+e_{j} e_{i}=-2 \delta_{i j}$. The resulting algebra, denoted by $\mathbb{R}_{n}$, is as a vector space isomorphic to the exterior algebra $\Lambda\left(\mathbb{R}^{n}\right)$ under the canonical isomorphism $e_{i_{1}} e_{i_{2}} \cdots e_{i_{k}} \mapsto$ $d x_{i_{1}} d x_{i_{2}} \cdots d x_{i_{k}}$, but it carries a richer multiplicative structure.

The Dirac operator is then defined by $\underline{\partial}=\sum_{i=1}^{n} e_{i} \partial_{x_{i}}$, and in view of the multiplication rules we indeed have that $\underline{\partial}^{2}=-\Delta_{n}$, which means that Clifford analysis is a refinement of harmonic analysis on $\mathbb{R}^{n}$. Since the operator $\underline{\partial}$ is invariant under rotations, Clifford analysis is perfectly suited for studying the orthogonal group $\mathrm{SO}(n)$, and in particular its double covering group $\operatorname{Spin}(n)$ (see

(c) A K Peters, Ltd. $1058-6458 / 2007 \$ 0.50$ per page Experimental Mathematics 16:1, page 55 
[Brackx et al. 82, Delanghe et al. 92, Gilbert and Murray 91]).

In [Sabadini and Sommen 02] and [Brackx et al. 05], the concept of Clifford analysis in the Hermitian setting was introduced. The main idea is to start from an evendimensional space $\mathbb{R}^{2 n}$ and to rewrite the vector variable in the following way:

$\underline{x}=\sum_{j=1}^{n}\left(e_{j} x_{j}+e_{j+n} x_{j+n}\right)=\sum_{j=1}^{n}\left(e_{j} x_{j}-i\left(i e_{j+n}\right) x_{j+n}\right)$.

If we set $x_{j+n}=y_{j}$ and $i e_{j+n}=\epsilon_{j}$, and introducing the Witt basis $\left\{\mathfrak{f}_{j}, \mathfrak{f}_{j}^{\dagger}\right\}(j=1, \ldots, m)$,

$$
\mathfrak{f}_{j}=\frac{e_{j}-\epsilon_{j}}{2} \text { and } \mathfrak{f}_{j}^{\dagger}=\frac{e_{j}+\epsilon_{j}}{2},
$$

this then gives rise to

$\underline{x}=\sum_{j=1}^{n}\left(\mathfrak{f}_{j}-\mathfrak{f}_{j}^{\dagger}\right) x_{j}+i\left(\mathfrak{f}_{j}+\mathfrak{f}_{j}^{\dagger}\right) y_{j}=\sum_{j=1}^{n}\left(f_{j} z_{j}-\mathfrak{f}_{j}^{\dagger} \bar{z}_{j}\right)=\underline{z}-\underline{z}^{\dagger}$.

Note that both sets $\left\{\mathfrak{f}_{j}\right\}_{j}$ and $\left\{\mathfrak{f}_{j}^{\dagger}\right\}_{j}$ generate a maximal isotropic $n$-dimensional subspace of $\mathbb{R}^{2 n}$, and that the Witt vectors satisfy the following multiplication rules:

$$
\mathfrak{f}_{j} \mathfrak{f}_{k}+\mathfrak{f}_{k} \mathfrak{f}_{j}=0=\mathfrak{f}_{j}^{\dagger} \mathfrak{f}_{k}^{\dagger}+\mathfrak{f}_{k}^{\dagger} \mathfrak{f}_{j}^{\dagger} \text { and } \quad \mathfrak{f}_{j} \mathfrak{f}_{k}^{\dagger}+\mathfrak{f}_{k}^{\dagger} \mathfrak{f}_{j}=\delta_{j k} .
$$

These Witt-basis vectors lead to an interesting characterization of the spinor space $S_{2 n}$ (the so-called Fock realization), which arises in a natural way as an irreducible representation space for the simple complex Clifford algebra $\mathbb{C}_{2 n}$. For that purpose, it suffices to define the idempotent $I=I_{1} \cdots I_{n}$, where $I_{j}=\mathfrak{f}_{j} \mathfrak{f}_{j}^{\dagger}$, and to put $S_{2 n}=\operatorname{Alg}_{\mathbb{C}}\left(\mathfrak{f}_{1}^{\dagger}, \ldots, \mathfrak{f}_{n}^{\dagger}\right) I=\mathbb{C} \Lambda_{n}^{\dagger} I$. We have hereby defined the (Grassmann) algebra $\mathbb{C} \Lambda_{n}^{\dagger}$, which will be referred to below. The subspace generated by products of $k$ elements will be denoted by $\left(\mathbb{C} \Lambda_{n}^{\dagger}\right)^{k}$.

In a completely similar way, we also have

$$
\begin{aligned}
\underline{\partial} & =\sum_{j=1}^{n}\left(\mathfrak{f}_{j}-\mathfrak{f}_{j}^{\dagger}\right) \partial_{x_{j}}+i\left(\mathfrak{f}_{j}+\mathfrak{f}_{j}^{\dagger}\right) \partial_{y_{j}} \\
& =2 \sum_{j=1}^{n}\left(\mathfrak{f}_{j} \partial_{\bar{z}_{j}}-\mathfrak{f}_{j}^{\dagger} \partial_{z_{j}}\right)=2\left(\underline{\partial}_{z}-\underline{\partial}_{z}^{\dagger}\right) .
\end{aligned}
$$

In Hermitian Clifford analysis, one then studies the firstorder differential operators $\underline{\partial}_{z}$ and $\underline{\partial}_{z}^{\dagger}$, commuting with the action of the unitary group on functions $f\left(\underline{z}, \underline{z}^{\dagger}\right)$. In view of the fact that

$$
\begin{aligned}
\left\{\underline{\partial}_{z}, \underline{\partial}_{z}^{\dagger}\right\} & =\underline{\partial}_{z} \underline{\partial}_{z}^{\dagger}+\underline{\partial}_{z}^{\dagger} \underline{\partial}_{z}=\Delta_{z}=\sum_{j} \partial_{z_{j}} \partial_{\bar{z}_{j}}=\frac{1}{4} \Delta_{2 n} \\
& =\frac{1}{4} \sum_{j=1}^{n}\left(\partial_{x_{j}}^{2}+\partial_{y_{j}}^{2}\right),
\end{aligned}
$$

the associated function theory leads to a refinement of harmonic analysis. To some extent, these operators were studied in [Rocha-Chavez et al. 01] using the language of forms. Part of the calculus was developed in [Sabadini and Sommen 02], with an emphasis on the calculation of resolutions of complexes in several complex variables. In [Brackx et al. 05] the authors initiated the systematic development of a basic function theory for the Hermitian version of Clifford analysis, in the special Euclidean setting.

In this paper we determine the building blocks for the Fischer decomposition in the Hermitian setting. In order to underline the importance of knowing these building blocks, we recall that in the classical orthogonal case the Fischer decomposition of an arbitrary homogeneous polynomial is completely determined in terms of the Fischer components of the reproducing kernel; see [Delanghe et al. 92].

The starting point for the Hermitian Fischer decomposition is again a reproducing kernel

$$
\mathcal{R}_{k, l}\left(\underline{z}, \underline{z}^{\dagger} ; \underline{u}, \underline{u}^{\dagger}\right)=\frac{\left\{\underline{z}, \underline{u}^{\dagger}\right\}^{k}\left\{\underline{z}^{\dagger}, \underline{u}\right\}^{l}}{k ! l !},
$$

in terms of which any $(k, l)$-homogeneous polynomial $R_{k, l}\left(\underline{z}, \underline{z}^{\dagger}\right)$ can be written using a Fischer-type inner product. As for the orthogonal case, it would be of great advantage to know the Fischer decomposition of the (scalar) reproducing kernel $\mathcal{R}_{k, l}$, since this would allow one to calculate the Hermitian Fischer decomposition of an arbitrary $(k, l)$-homogeneous polynomial $R_{k, l}\left(\underline{z}, \underline{z}^{\dagger}\right)$ in terms of (Hermitian) zonal building blocks $Z_{k, l}\left(\underline{z}, \underline{z}^{\dagger} ; \underline{u}, \underline{u}^{\dagger}\right)$.

An explicit form for these building blocks for the Hermitian Fischer decomposition of the kernel was formulated as a claim in [Brackx et al. 05, Sections 3 through 5], and the aim of this paper is to investigate this claim. This is the first step toward explicit formulas for the Hermitian Fischer decomposition.

\section{SPIN-EULER POLYNOMIALS OF TYPES $(k, 0)$ AND $(0, k)$}

Throughout this paper, we will constantly encounter the following specific Clifford number:

Definition 2.1. The spin-Euler operator $\beta$ is defined as

$$
\beta=\underline{\partial}_{z}[\underline{z}]=\sum_{j} f_{j}^{\dagger} f_{j}=\frac{n}{2}+\frac{i}{2} \sum_{j=1}^{n} e_{j} e_{j+n} .
$$


This Clifford number is called an operator, because its action (through multiplication) on a spinor $\psi \in\left(\mathbb{C} \Lambda_{n}^{\dagger}\right)^{k} I$ is given by $\beta[\psi]=k \psi$ (for all $k=1, \ldots, n$ ).

In view of the fact that $\mathbb{C} \Lambda_{n}^{\dagger}=\bigoplus_{j=0}^{n}\left(\mathbb{C} \Lambda_{n}^{\dagger}\right)^{k}$, it is immediately clear that this operator satisfies the equation $\beta(\beta-1) \cdots(\beta-n)=0$. This polynomial expression in $\beta$ expresses the fact that the spin-Euler operator can be considered as a root of the so-called falling factorial function $(x)_{k}$ defined for all $k \in \mathbb{N}$ as $(x)_{k}=$ $x(x-1)(x-2) \cdots(x-k+1)$; see, for example, [Graham et al. 94].

They generate the Stirling numbers of the first kind $s(k, j)$ by means of $(x)_{k}=\sum_{j=0}^{k} s(k, j) x^{j}$. The Stirling number of the first kind is usually defined as the number $s(k, j)$ such that the number of permutations of $k$ elements containing exactly $j$ permutation cycles is precisely the nonnegative number $|s(k, j)|=(-1)^{k-j} s(k, j)$. This means that $s(k, j)=0$ for $j>k, s(k, k)=1$, and $s(k, 0)=\delta_{k, 0}$.

In the case $(k, l)=(k, 0)$, the expression that was proposed in [Brackx et al. 05] reduces to

$$
Z_{k, 0}\left(\underline{z}, \underline{z}^{\dagger} ; \underline{u}, \underline{u}^{\dagger}\right)=\frac{\left\{\underline{z}, \underline{u}^{\dagger}\right\}^{k}}{k !}+\frac{\underline{z} P_{k}(\beta)\left\{\underline{z}, \underline{u}^{\dagger}\right\}^{k-1} \underline{u}^{\dagger}}{(k-1) !},
$$

where $P_{k}(\beta)$ denotes a polynomial of degree $n$ in $\beta$. We call this polynomial, depending on $k \in \mathbb{N}$, the spin-Euler polynomial of type $(k, 0)$. To determine this polynomial, we make use of the monogeneity conditions for the Hermitian building blocks $Z_{k, 0}\left(\underline{z}, \underline{z}^{\dagger} ; \underline{u}, \underline{u}^{\dagger}\right)$. Since these depend only on $\underline{z}$ and $\underline{u}^{\dagger}$, we obtain $\underline{\partial}_{z} Z_{k, 0}=0=Z_{k, 0} \underline{\partial}_{u}^{\dagger}$. It is easily verified that this gives rise to the equation

$$
\frac{1}{(k-1) !}\left(1+(k-1+\beta) P_{k}(\beta)\right) \underline{u}^{\dagger} \underline{z} \underline{u}^{\dagger}\left\{\underline{z}, \underline{u}^{\dagger}\right\}^{k-2}=0
$$

where we have made use of the following lemma, which is easily proved by induction:

Lemma 2.2. If $P(\beta)$ denotes a polynomial in $\beta$, we have $\left[P(\beta), \underline{u}^{\dagger} \underline{z}\right]=0$. This remains true for any combination of a complex variable and the Hermitian conjugate of a complex variable. We thus also have that (for example)

$$
\left[P(\beta), \underline{z} \wedge \underline{z}^{\dagger}\right]=\left[P(\beta), \underline{u} \wedge \underline{u}^{\dagger}\right]=0 \text {. }
$$

Note that we restrict ourselves to the cases $k>1$ in the equation for $P_{k}(\beta)$.

In the case $k=1$ it suffices to note that

$$
(\beta)_{n+1}=0 \Longrightarrow\left((-1)^{n} n !+\beta P_{1}(\beta)\right) \beta=0 .
$$

In general, the zero divisor $\beta$ cannot be divided out, unless we multiply the right-hand side by $\underline{u}^{\dagger}$, leading to

$$
\left((-1)^{n} n !+\beta P_{1}(\beta)\right) \underline{u}^{\dagger}(\beta+1)=0 \text {. }
$$

Remark 2.3. The condition $Z_{k, 0} \underline{\partial}_{u}^{\dagger}=0$ leads to the same equations for $P_{k}(\beta)$, in view of the symmetry. This remains true in general: in view of the symmetry of $Z_{k, l}\left(\underline{z}, \underline{z}^{\dagger} ; \underline{u}, \underline{u}^{\dagger}\right)$ with respect to $\left(\underline{z}, \underline{u}^{\dagger}\right)$ and $\left(\underline{z}^{\dagger}, \underline{u}\right)$, the defining relations for the spin-Euler polynomials will always follow from monogeneity conditions on $\underline{z}$ and $\underline{z}^{\dagger}$ only.

In view of the claim, we then put $P_{k}(\beta)=$ $\sum_{j=0}^{n} c_{j}^{(k)} \beta^{j}$, where the coefficients $c_{j}^{(k)}$ are to be determined. Plugging this polynomial into $(2-1)$, we are led to the following equation:

$$
1+(k-1) \sum_{j=0}^{n} c_{j}^{(k)} \beta^{j}+\sum_{j=0}^{n} c_{j}^{(k)} \beta^{j+1}=0
$$

This looks like an overdetermined system, since we now have $(n+2)$ equations for only $(n+1)$ unknowns, but from the fact that $(\beta)_{m}=0$ it follows that $\beta^{n+1}=$ $-\sum_{j=0}^{n} s(n+1, j) \beta^{j}$, whence Equation $(2-1)$ reduces to $1+\sum_{j=0}^{n}\left((k-1) c_{j}^{(k)}-s(n+1, j) c_{n}^{(k)}\right) \beta^{j}+\sum_{j=1}^{n} c_{j-1}^{(k)} \beta^{j}=0$.

Arranging this equation into the matrix formalism, we get $M C=R$ with

$$
M=\left(\begin{array}{cccccc}
k-1 & 0 & 0 & 0 & \cdots & -s(n+1,0) \\
1 & k-1 & 0 & 0 & \cdots & -s(n+1,1) \\
0 & 1 & k-1 & 0 & \cdots & -s(n+2,0) \\
0 & 0 & 1 & k-1 & \cdots & -s(n+3,0) \\
\vdots & \vdots & \vdots & \vdots & \ddots & \vdots \\
0 & 0 & 0 & 0 & \cdots & k-1-s(n+1, n)
\end{array}\right)
$$

with $C=\left(c_{0}^{(k)}, \ldots, c_{n}^{(k)}\right)^{T}$ and $R=(-1,0, \ldots, 0)^{T}$. The existence of the spin-Euler polynomial $P_{k}(\beta)$ thus depends on the determinant of the matrix $M$, for which we refer to the following lemma:

Lemma 2.4. The determinant of the matrix $M$ is given by

$$
|M|=\sum_{j=0}^{n+1}|s(n+1, j)|(k-1)^{j}
$$

Proof: The result follows from an induction argument. 
Corollary 2.5. For all $k>1$, the matrix $M$ is invertible. From this the existence of the spin-Euler polynomial of type $(k, 0)$, with $k \in \mathbb{N}_{0}$, immediately follows.

In order to obtain an expression for $P_{k}(\beta)$, it suffices to invert the matrix $M$. In view of the fact that we will multiply $M^{-1}$ by $R$, it suffices to determine only the first column of this matrix. This is the subject of the following lemma, which again follows from a straightforward induction argument.

Lemma 2.6. The first column of the matrix $M^{-1}$ is given by

$$
\frac{1}{|M|}\left(\mu^{-1}\right)_{j, 1} \quad \text { with } \quad j \in\{1, \ldots, n+1\},
$$

where

$$
\mu_{j, 1}^{-1}=(-1)^{j-1} \sum_{i=j}^{n+1}|s(n+1, i)|(k-1)^{i-j} .
$$

This then leads to the following theorem.

Theorem 2.7. The zonal Hermitian complex polynomials $Z_{k, 0}\left(\underline{z}, \underline{z}^{\dagger} ; \underline{u}, \underline{u}^{\dagger}\right)$ are given by

$$
Z_{k, 0}\left(\underline{z}, \underline{z}^{\dagger} ; \underline{u}, \underline{u}^{\dagger}\right)=\frac{\left\{\underline{z}, \underline{u}^{\dagger}\right\}^{k}}{k !}+\frac{\underline{z} P_{k}(\beta)\left\{\underline{z}, \underline{u}^{\dagger}\right\}^{k-1} \underline{u}^{\dagger}}{(k-1) !},
$$

where the spin-Euler polynomials $P_{k}(\beta)$ of type $(k, 0)$ are defined as follows:

$P_{1}(\beta)=\frac{(-1)^{n}}{n !} \sum_{j=0}^{n-1} s(n+1, j+2) \beta^{j}$,
$P_{k}(\beta)=\sum_{j=1}^{n+1}(-1)^{j}\left(\frac{\sum_{i=j}^{n+1}|s(n+1, i)|(k-1)^{i-j}}{\sum_{i=0}^{n+1}|s(n+1, i)|(k-1)^{i}}\right) \beta^{j-1}$,

$k>1$. These polynomials solve the equations

$$
\begin{aligned}
\left(1+\beta P_{1}(\beta)\right) \underline{u}^{\dagger} & =0, \\
\left(1+(k-1+\beta) P_{k}(\beta)\right) & =0 \quad(k>1) .
\end{aligned}
$$

Note that in the case $k=1$, the factor $\underline{u}^{\dagger}$ cannot be omitted.

In order to determine the spin-Euler polynomials of type $(0, k)$, which we will denote by $P^{k}(\beta)$, it suffices to note that up to the subtle difference between the variables $\underline{z}$ and $\underline{z}^{\dagger}$ encoded in the equations

$$
\underline{\partial}_{z}[\underline{z}]=\beta \longleftrightarrow \underline{\partial}_{z}^{\dagger}\left[\underline{z}^{\dagger}\right]=n-\beta,
$$

the defining equations will be completely similar. This immediately leads to the following conclusion:

Theorem 2.8. The zonal Hermitian complex polynomials $Z_{0, k}\left(\underline{z}, \underline{z}^{\dagger} ; \underline{u}, \underline{u}^{\dagger}\right)$ are given by

$$
Z_{0, k}\left(\underline{z}, \underline{z}^{\dagger} ; \underline{u}, \underline{u}^{\dagger}\right)=\frac{\left\{\underline{z}^{\dagger}, \underline{u}\right\}^{k}}{k !}+\frac{\underline{z}^{\dagger} P^{k}(\beta)\left\{\underline{z^{\dagger}}, \underline{u}\right\}^{k-1} \underline{u}}{(k-1) !},
$$

where the spin-Euler polynomials $P^{k}(\beta)$ of type $(0, k)$ are defined by $P^{k}(\beta)=P_{k}(n-\beta)$. These polynomials solve the equations

$$
\begin{aligned}
\left(1+(n-\beta) P^{1}(\beta)\right) \underline{u} & =0, \\
\left(1+(k-1+n-\beta) P^{k}(\beta)\right) & =0 \quad(k>1) .
\end{aligned}
$$

\section{COMMUTATION BEHAVIOR OF SPIN-EULER POLYNOMIALS}

In this section, we investigate the way in which a polynomial in $\beta$ behaves under commutation with a complex variable or the Hermitian conjugate of a complex variable.

Lemma 3.1. For all $j \in \mathbb{N}$, we have

$$
\begin{aligned}
{\left[\underline{u}, \beta^{j+1}\right] } & =\sum_{k=0}^{j} \beta^{k}(1+\beta)^{j-k} \underline{u}=\sum_{l=0}^{j}\left\{\sum_{i=0}^{l}\left(\begin{array}{l}
j-i \\
l-i
\end{array}\right)\right\} \beta^{l} \underline{u} \\
& =\sum_{l=0}^{j}\left(\begin{array}{c}
j+1 \\
l
\end{array}\right) \beta^{l} \underline{u} .
\end{aligned}
$$

In this expression, $\underline{u}$ may be replaced by any complex variable.

Proof: The first statement is easily proved by induction on the power $j$. In order to prove the second equality, it suffices to note that if we write

$$
\sum_{k=0}^{j} \beta^{k}(1+\beta)^{j-k}=\sum_{l=0}^{j} c_{l} \beta^{l},
$$

there is a contribution to each power $\beta^{l}$ from all $\beta^{k}(1+$ $\beta)^{j-k}$ with $k \leq l$. This contribution, which is the coefficient of $\beta^{l-k}$ in $(1+\beta)^{j-k}$, is given by $\left(\begin{array}{c}j-k \\ l-k\end{array}\right)$. To prove the third equality, we use induction on the parameter $l$.

Corollary 3.2. If $P(\beta)=\sum_{j=0}^{n} c_{j} \beta^{j}$ denotes a polynomial of degree $n$ in $\beta$, the commutator of this polynomial with 
$\underline{u}$ is given by

$$
[\underline{u}, P(\beta)]=\sum_{j=1}^{n} c_{j}\left[\underline{u}, \beta^{j}\right]=\sum_{i=0}^{n-1}\left(\sum_{j=i+1}^{n}\left(\begin{array}{l}
j \\
i
\end{array}\right) c_{j}\right) \beta^{i} \underline{u} .
$$

In what follows, we will denote the coefficients of this polynomial by $\gamma_{i}^{(c)}$. The index $c$ refers here to the fact that these coefficients $\gamma_{i}$ depend on the coefficients $c_{j}$ of the given polynomial. Because we will often encounter the commutator of a (spin-Euler) polynomial with a variable $\underline{u}$ (or $\underline{z}$ ) in what follows, we introduce the following notation:

Definition 3.3. If a polynomial $P(\beta)=\sum_{j=0}^{n} c_{j} \beta^{j}$ is commuted with a complex variable (for example $\underline{u}$ or $\underline{z}$ ), this gives rise to a polynomial of degree $(n-1)$ in $\beta$, denoted by

$$
[P](\beta)=\sum_{i=0}^{n-1} \gamma_{i}^{(c)} \beta^{i}=\sum_{i=0}^{n-1}\left(\sum_{j=i+1}^{n}\left(\begin{array}{l}
j \\
i
\end{array}\right) c_{j}\right) \beta^{i} .
$$

We thus, for example, have that $[\underline{u}, P(\beta)]=[P](\beta) \underline{u}$.

In a similar way, the following lemma can be proved:

Lemma 3.4. For all $j \in \mathbb{N}$ we have

$$
\begin{aligned}
{\left[\underline{u}^{\dagger}, \beta^{j+1}\right] } & =-\sum_{l=0}^{j}(-1)^{l+j}\left\{\sum_{i=0}^{l}\left(\begin{array}{c}
j+i-l \\
i
\end{array}\right)\right\} \beta^{l} \underline{u}^{\dagger} \\
& =(-1)^{j+1} \sum_{l=0}^{j}(-1)^{l}\left(\begin{array}{c}
j+1 \\
l
\end{array}\right) \beta^{l} \underline{u}^{\dagger} .
\end{aligned}
$$

A similar expression is of course obtained if $\underline{u}^{\dagger}$ is replaced by the Hermitian conjugate of any complex variable.

Corollary 3.5. If $P(\beta)=\sum_{j=0}^{n} c_{j} \beta^{j}$ denotes a polynomial of degree $n$ in $\beta$, the commutator of this polynomial with $\underline{u}^{\dagger}$ is given by

$$
\begin{aligned}
{\left[\underline{u}^{\dagger}, P(\beta)\right] } & =\sum_{j=1}^{n} c_{j}\left[\underline{u}^{\dagger}, \beta^{j}\right] \\
& =\sum_{i=0}^{n-1}\left(\sum_{j=i+1}^{n}(-1)^{i+j}\left(\begin{array}{l}
j \\
i
\end{array}\right) c_{j}\right) \beta^{i} \underline{u}^{\dagger}
\end{aligned}
$$

In what follows, we will denote the coefficients of this polynomial by $\delta_{i}^{(c)}$. The index $c$ refers to the fact that these coefficients depend on the coefficients $c_{j}$ of the given polynomial. Again in view of the fact that we will often encounter the commutator of a polynomial in $\beta$ with a conjugated variable $\underline{u}^{\dagger}$ (or $\underline{z}^{\dagger}$ ) in what follows, we introduce the following notation:

Definition 3.6. If a polynomial $P(\beta)=\sum_{j=0}^{n} c_{j} \beta^{j}$ is commuted with the Hermitian conjugate of a complex variable (for example $\underline{u}^{\dagger}$ or $\underline{z}^{\dagger}$ ), this gives rise to a polynomial of degree $(n-1)$ in $\beta$, denoted by

$$
[P]^{\dagger}(\beta)=\sum_{i=0}^{n-1} \delta_{i}^{(c)} \beta^{i}=\sum_{i=0}^{n-1}\left(\sum_{j=i+1}^{n}(-1)^{i+j}\left(\begin{array}{l}
j \\
i
\end{array}\right) c_{j}\right) \beta^{i} .
$$

We thus, for example, have that

$$
\left[\underline{u}^{\dagger}, P(\beta)\right]=[P]^{\dagger}(\beta) \underline{u}^{\dagger} .
$$

Note that in view of the fact that $\beta^{\dagger}=\beta$, there is no need to consider the Hermitian conjugate of the polynomial $[P](\beta)$ defined earlier, whence the notation $[P]^{\dagger}(\beta)$ is unambiguous.

From the previous lemmas, we easily prove the following lemma:

Lemma 3.7. If $P(\beta)$ denotes an arbitrary polynomial in $\beta$, we have

$$
[P(\beta), \underline{u}]=\underline{u}[P]^{\dagger}(\beta) \quad \text { and } \quad\left[P(\beta), \underline{u}^{\dagger}\right]=\underline{u}^{\dagger}[P](\beta) .
$$

Lemma 3.8. For all $j \in \mathbb{N}$, we have

$$
\begin{aligned}
{\left[\underline{u},(n-\beta)^{j+1}\right] } & =-\sum_{k=0}^{j}(n-\beta)^{j-k}(n-\beta-1)^{k} \underline{u} \\
& =(-1)^{j+1} \sum_{l=0}^{j}(-1)^{l}\left(\begin{array}{c}
j+1 \\
l
\end{array}\right)(n-\beta)^{l} \underline{u}, \\
{\left[\underline{u}^{\dagger},(n-\beta)^{j+1}\right] } & =\sum_{k=0}^{j}(n-\beta)^{k}(1+n-\beta)^{j-k} \underline{u}^{\dagger} \\
& =\sum_{l=0}^{j}\left(\begin{array}{c}
j+1 \\
l
\end{array}\right)(n-\beta)^{l} \underline{u}^{\dagger},
\end{aligned}
$$

where $\underline{u}$ and $\underline{u}^{\dagger}$ may of course be replaced by $\underline{z}$ and $\underline{z}^{\dagger}$.

Corollary 3.9. If $P(\beta)=\sum_{j=0}^{n} c_{j} \beta^{j}$ denotes a polynomial of degree $n$ in $\beta$, the previous lemma can be rewritten in such a way that the duality between $\beta$ and $(n-\beta)$ may be observed from the following commutators:

$$
\begin{gathered}
{[\underline{u}, P(\beta)]=[P](\beta) \underline{u} \longleftrightarrow[\underline{u}, P(n-\beta)]=[P]^{\dagger}(n-\beta) \underline{u},} \\
{\left[\underline{u}^{\dagger}, P(\beta)\right]=[P]^{\dagger}(\beta) \underline{u}^{\dagger} \longleftrightarrow\left[\underline{u}^{\dagger}, P(n-\beta)\right]=[P](n-\beta) \underline{u}^{\dagger} .}
\end{gathered}
$$


To end this section, we also mention the following result.

Lemma 3.10. If $P(\beta)$ denotes an arbitrary polynomial in $\beta$, we have

$$
\begin{aligned}
{[P(n-\beta), \underline{u}] } & =\underline{u}[P](n-\beta), \\
{\left[P(n-\beta), \underline{u}^{\dagger}\right] } & =\underline{u}^{\dagger}[P]^{\dagger}(n-\beta) .
\end{aligned}
$$

\section{SPIN-EULER POLYNOMIALS OF TYPE $(k, k)$}

\subsection{A First Special Case: $k=1$}

We begin this section with the calculations for $Z_{1,1}\left(\underline{z}, \underline{z}^{\dagger} ; \underline{u}, \underline{u}^{\dagger}\right)$ in order to illustrate how the case in which both $k$ and $l$ are nonzero differs substantially from the previous calculations. By definition, we put

$$
\begin{aligned}
Z_{1,1}\left(\underline{z}, \underline{z}^{\dagger} ; \underline{u}, \underline{u}^{\dagger}\right) \\
=\left\{\underline{z}, \underline{u}^{\dagger}\right\}\left\{\underline{z}^{\dagger}, \underline{u}\right\} \\
+\left(\underline{z} P_{1,1}^{(1)}(\beta)\left\{\underline{z}^{\dagger}, \underline{u}\right\} \underline{u}^{\dagger}+\underline{z}^{\dagger} P_{1,1}^{(2)}(\beta)\left\{\underline{z}, \underline{u}^{\dagger}\right\} \underline{u}\right) \\
+\left(\underline{z} \wedge \underline{z}^{\dagger} P_{1,1}^{(3)}(\beta) \underline{u} \wedge \underline{u}^{\dagger}+\underline{z} \wedge \underline{z}^{\dagger} P_{1,1}^{(4)}(\beta)|\underline{u}|^{2}\right. \\
\left.\quad+|\underline{z}|^{2} P_{1,1}^{(5)}(\beta) \underline{u} \wedge \underline{u}^{\dagger}\right) \\
\quad+|\underline{z}|^{2} P_{1,1}^{(6)}(\beta)|\underline{u}|^{2},
\end{aligned}
$$

where all the polynomials $P_{1,1}^{(i)}(\beta)$, with $i \in\{1, \ldots, 6\}$, are to be determined. The monogeneity conditions for $Z_{1,1}$ will give rise to six equations. In order to derive these, we use the following lemma:

Lemma 4.1. The variables $\underline{z}$ and $\underline{z}^{\dagger}$ satisfy the following relations:

$$
\underline{\partial}_{z} \underline{z} \wedge \underline{z}^{\dagger}=\left(\beta-\frac{1}{2}\right) \underline{z}^{\dagger}
$$

and

$$
\underline{\partial}_{z}^{\dagger} \underline{z} \wedge \underline{z}^{\dagger}=\left(\beta+\frac{1}{2}-n\right) \underline{z} .
$$

The actions of $\underline{\partial}_{z}$ and $\underline{\partial}_{z}^{\dagger}$ give rise to the following terms:

$$
\begin{array}{r}
\underline{\partial}_{z} \rightarrow \underline{u}^{\dagger}\left\{\underline{z}^{\dagger}, \underline{u}\right\}+\left\{\begin{array}{l}
\beta P_{1,1}^{(1)}\left\{\underline{z}^{\dagger}, \underline{u}\right\} \underline{u}^{\dagger} \\
\underline{u}^{\dagger} \underline{z}^{\dagger} P_{1,1}^{(2)} \underline{u}
\end{array}\right. \\
+\left\{\begin{array}{l}
\left(\beta-\frac{1}{2}\right) \underline{z}^{\dagger} P_{1,1}^{(3)} \underline{u} \wedge \underline{u}^{\dagger} \\
\left(\beta-\frac{1}{2}\right) \underline{z}^{\dagger} P_{1,1}^{(4)}|\underline{u}|^{2} \\
\underline{z}^{\dagger} P_{1,1}^{(5)} \underline{u} \wedge \underline{u}^{\dagger} \\
\underline{z}^{\dagger} P_{1,1}^{(6)}|\underline{u}|^{2}
\end{array}\right.
\end{array}
$$

and

$$
\begin{aligned}
\underline{\partial}_{z}^{\dagger} \rightarrow & \underline{u}\left\{\underline{z}, \underline{u}^{\dagger}\right\}+\left\{\begin{array}{l}
(n-\beta) P_{1,1}^{(2)}\left\{\underline{z}, \underline{u}^{\dagger}\right\} \underline{u} \\
\underline{u} \underline{z} P_{1,1}^{(1)} \underline{u}^{\dagger}
\end{array}\right. \\
+ & \left\{\begin{array}{l}
\left(\beta+\frac{1}{2}-n\right) \underline{z} P_{1,1}^{(3)} \underline{u} \wedge \underline{u}^{\dagger} \\
\left(\beta+\frac{1}{2}-n\right) \underline{z} P_{1,1}^{(4)}|\underline{u}|^{2} \\
\underline{z} P_{1,1}^{(5)} \underline{u} \wedge \underline{u}^{\dagger} \\
\underline{z} P_{1,1}^{(6)}|\underline{u}|^{2}
\end{array}\right.
\end{aligned}
$$

Apart from the terms $\underline{u}^{\dagger} \underline{z}^{\dagger} P_{1,1}^{(2)} \underline{u}$ and $\underline{u} \underline{z} P_{1,1}^{(1)} \underline{u}^{\dagger}$, it is immediately clear how to arrange these terms into a system of six equations. These two exceptional terms will give contributions to the equations in $\underline{z}^{\dagger}\left(\underline{u} \wedge \underline{u}^{\dagger}\right)$ and $\underline{z}^{\dagger}|\underline{u}|^{2}$ (and similar terms with $\underline{z}$ ). This eventually gives rise to the following equations:

$$
\begin{array}{r}
\left(1+\beta P_{1,1}^{(1)}\right) \underline{u}^{\dagger}\left\{\underline{z}^{\dagger}, \underline{u}\right\}=0, \\
\left(1+(n-\beta) P_{1,1}^{(2)}\right) \underline{u}\left\{\underline{z}, \underline{u}^{\dagger}\right\}=0, \\
\underline{z}^{\dagger}\left(\left(\beta+\frac{1}{2}\right) P_{1,1}^{(3)}+P_{1,1}^{(5)}+P_{1,1}^{(2)}+\left[P_{1,1}^{(2)}\right]^{+}\right) \underline{u} \wedge \underline{u}^{\dagger}=0, \\
\underline{z}\left(\left(\beta-\frac{1}{2}-n\right) P_{1,1}^{(3)}+P_{1,1}^{(5)}-P_{1,1}^{(1)}-\left[P_{1,1}^{(1)}\right]\right) \underline{u} \wedge \underline{u}^{\dagger}=0, \\
\underline{z}^{\dagger}\left(\left(\beta+\frac{1}{2}\right) P_{1,1}^{(4)}+P_{1,1}^{(6)}-\frac{1}{2} P_{1,1}^{(2)}-\frac{1}{2}\left[P_{1,1}^{(2)}\right]^{+}\right)|\underline{u}|^{2}=0, \\
\underline{z}\left(\left(\beta-\frac{1}{2}-n\right) P_{1,1}^{(4)}+P_{1,1}^{(6)}-\frac{1}{2} P_{1,1}^{(1)}-\frac{1}{2}\left[P_{1,1}^{(1)}\right]\right)|\underline{u}|^{2}=0 .
\end{array}
$$

It follows immediately that $P_{1,1}^{(1)}(\beta)=P_{1}(\beta)$ and $P_{1,1}^{(2)}(\beta)=P^{1}(\beta)$. Before determining the other polynomials, we first prove the following lemma.

Lemma 4.2. For each $k \in \mathbb{N}$ we have

$$
P_{k}(\beta)+\left[P_{k}\right](\beta)=P_{k+1}(\beta)
$$

and

$$
P^{k}(\beta)+\left[P^{k}\right]^{\dagger}(\beta)=P^{k+1}(\beta)
$$

Proof: It suffices to observe that

$$
\begin{aligned}
(1+ & \left.(\beta+k-1) P_{k}\right) \underline{u}^{\dagger}=0 \\
& \Longleftrightarrow \underline{u}^{\dagger}\left(1+(\beta+k)\left(P_{k}+\left[P_{k}\right]\right)\right)=0,
\end{aligned}
$$

together with the fact that there is a unique polynomial solution of degree $n$. The other statement is proved in a similar way.

Subtracting the third and fourth (respectively fifth and sixth) equations above, we obtain

$$
\begin{array}{r}
(n+1) P_{1,1}^{(3)}(\beta)+P_{2}(\beta)+P_{2}(n-\beta)=0 \\
\Longrightarrow P_{1,1}^{(3)}(\beta)=-\frac{P_{2}(\beta)+P_{2}(n-\beta)}{n+1},
\end{array}
$$


respectively

$$
\begin{gathered}
(n+1) P_{1,1}^{(4)}(\beta)-\frac{1}{2}\left(P_{2}(n-\beta)-P_{2}(\beta)\right)=0 \\
\Longrightarrow P_{1,1}^{(4)}(\beta)=\frac{P_{2}(n-\beta)-P_{2}(\beta)}{2(n+1)} .
\end{gathered}
$$

Plugging these expressions back into the third and fourth (fifth and sixth) equations, we finally obtain

$$
P_{1,1}^{(5)}(\beta)=-P_{2}(n-\beta)+\left(\beta+\frac{1}{2}\right) \frac{P_{2}(\beta)+P_{2}(n-\beta)}{n+1}
$$

and

$$
\begin{aligned}
& P_{1,1}^{(6)}(\beta) \\
& \quad=\frac{1}{2}\left(P_{2}(n-\beta)+\left(\beta+\frac{1}{2}\right) \frac{P_{2}(\beta)-P_{2}(n-\beta)}{(n+1)}\right) .
\end{aligned}
$$

\subsection{A Second Special Case: $k=2$}

Before turning to the most general case, it will be useful to consider $Z_{2,2}\left(\underline{z}, \underline{z}^{\dagger} ; \underline{u}, \underline{u}^{\dagger}\right)$ as a special case as well. The reason for this comes from the fact that the case $(2,2)$ leads to some peculiar features that could not be observed in the simpler case $(1,1)$. We first introduce the following definition:

Definition 4.3. For arbitrary $(a, b) \in \mathbb{N}^{2}$ we define

$$
S(a, b)=\frac{\left\{\underline{z}, \underline{u}^{\dagger}\right\}^{a}\left\{\underline{z}^{\dagger}, \underline{u}\right\}^{b}}{a ! b !},
$$

where $S$ refers to the fact that this factor is scalar.

Note that $\underline{\partial}_{z} S(a, b)=\underline{u}^{\dagger} S(a-1, b)$ and $\underline{\partial}_{z}^{\dagger} S(a, b)=$ $\underline{u} S(a, b-1)$, two relations that will be useful in what follows.

Let $Z_{2,2}\left(\underline{z}, \underline{z}^{\dagger} ; \underline{u}, \underline{u}^{\dagger}\right)$ be defined by

$$
\begin{aligned}
& |\underline{z}|^{0}|\underline{u}|^{0}\left(\begin{array}{c}
c S(2,2)+\left(\begin{array}{c}
z P_{2,2}^{(1)} S(1,2) \underline{u} \\
\dagger
\end{array}+\underline{z}^{\dagger} P_{2,2}^{(2)} S(2,1) \underline{u}\right) \\
S(1,1)\left(\underline{z} \wedge \underline{z}^{\dagger} P_{2,2}^{(3)} \underline{u} \wedge \underline{u}^{\dagger}+\underline{z} \wedge \underline{z}^{\dagger} P_{2,2}^{(4)}|\underline{u}|^{2}+|\underline{z}|^{2} P_{2,2}^{(5)} \underline{u} \wedge \underline{u}^{\dagger}\right)
\end{array}\right) \\
& |\underline{z}|^{2}|\underline{u}|^{2}\left(\begin{array}{c}
c P_{2,2}^{(6)} S(1,1)+\left(\underline{z}_{2,2}^{(7)} S(0,1) \underline{u} \underline{u}^{\dagger}+\underline{z}^{\dagger} P_{2,2}^{(8)} S(1,0) \underline{u}\right) \\
\left(\underline{z} \wedge \underline{z}^{\dagger} P_{2,2}^{(9)} \underline{u} \wedge \underline{u}^{\dagger}+\underline{z} \wedge \underline{z}^{\dagger} P_{2,2}^{(10)}|\underline{u}|^{2}+|\underline{z}|^{2} P_{2,2}^{(11)} \underline{u} \wedge \underline{u}^{\dagger}\right)
\end{array}\right) \\
& |\underline{z}|^{4}|\underline{u}|^{4} P_{2,2}^{(12)} .
\end{aligned}
$$

This time, there are 12 spin-Euler polynomials of type $(2,2)$ to be determined. To do so we have to rearrange all contributions coming from the action of $\underline{\partial}_{z}$ and $\underline{\partial}_{z}^{\dagger}$ into a system of equations. Let us work out the action of $\underline{\partial}_{z}$. The action of $\underline{\partial}_{z}^{\dagger}$ will give rise to similar terms. When acting on the zeroth row (by which we mean the terms multiplied by $|\underline{z}|^{0}|\underline{u}|^{0}$ ), we first of all get the following terms, which are more or less the analogues of the terms obtained for the case $(1,1)$ :

$$
\begin{aligned}
S(1,2) \underline{u}^{\dagger}+\left\{\begin{array}{l}
(1+\beta) P_{2,2}^{(1)} S(1,2) \underline{u}^{\dagger} \\
S(1,1) \underline{u}^{\dagger} \underline{z}^{\dagger} P_{2,2}^{(2)} \underline{u}
\end{array}\right. \\
+S(1,1)\left\{\begin{array}{l}
\underline{z}^{\dagger}\left(\beta+\frac{1}{2}\right) P_{2,2}^{(3)} \underline{u} \wedge \underline{u}^{\dagger} \\
\underline{z}^{\dagger}\left(\beta+\frac{1}{2}\right) P_{2,2}^{(4)}|\underline{u}|^{2} \\
\underline{z}^{\dagger} P_{2,2}^{(5)} \underline{u} \wedge \underline{u}^{\dagger}
\end{array}\right.
\end{aligned}
$$

However, in contrast to the case $(1,1)$, there is another contribution coming from the terms in the first row: this is due to the presence of the factor $S(1,1)$ in front of the second set of parentheses. These terms are given by

$\underline{u}^{\dagger} S(0,1)\left(\underline{z} \wedge \underline{z}^{\dagger} P_{2,2}^{(3)} \underline{u} \wedge \underline{u}^{\dagger}+\underline{z} \wedge \underline{z}^{\dagger} P_{2,2}^{(4)}|\underline{u}|^{2}+|\underline{z}|^{2} P_{2,2}^{(5)} \underline{u} \wedge \underline{u}^{\dagger}\right)$.

In view of the fact that

$$
\underline{u}^{\dagger} S(0,1)|\underline{z}|^{2} P_{2,2}^{(5)} \underline{u} \wedge \underline{u}^{\dagger}=\frac{1}{2}|\underline{z}|^{2}|\underline{u}|^{2} S(0,1) \underline{u} \underline{u}^{\dagger} P_{2,2}^{(5)},
$$

the last term will contribute to equations coming from the action of $\underline{\partial}_{z}$ acting on the first row (this will become clear in a moment). The two remaining terms are new, when compared with the case $(1,1)$, and will give rise to additional equations. First of all, note that they are not independent, for the simple reason that

$$
\begin{aligned}
\underline{u}^{\dagger}(\underline{z} & \left.\wedge \underline{z}^{\dagger}\right)\left(\underline{u} \wedge \underline{u}^{\dagger}\right) \\
& =\frac{1}{2} \underline{u}^{\dagger}|\underline{u}|^{2} \underline{z} \wedge \underline{z}^{\dagger}-S(1,0) \underline{z}^{\dagger}\left(\frac{1}{2}|\underline{u}|^{2}-\underline{u} \wedge \underline{u}^{\dagger}\right) .
\end{aligned}
$$

In other words, besides another contribution to $S(1,1) \underline{z}^{\dagger} \underline{u} \wedge \underline{u}^{\dagger}$ and $S(1,1) \underline{z}^{\dagger}|\underline{u}|^{2}$, there are also terms

$$
S(0,1) \underline{u} \underline{u}^{\dagger}|\underline{u}|^{2} \underline{z} \wedge \underline{z}^{\dagger}\left(\frac{1}{2} P_{2,2}^{(3)}+P_{2,2}^{(4)}\right) .
$$

Next, we consider the action of $\underline{\partial}_{z}$ on the first row. The obvious contribution is now given by

$$
\begin{gathered}
|\underline{z}|^{2}|\underline{u}|^{2}\left(S(0,1) \underline{u}^{\dagger} P_{2,2}^{(6)}+\left\{\begin{array}{l}
\beta P_{2,2}^{(7)} S(0,1) \underline{u}^{\dagger} \\
\underline{u}^{\dagger} \underline{z}^{\dagger} P_{2,2}^{(8)} \underline{u}
\end{array}\right.\right. \\
+\left\{\begin{array}{l}
\underline{z}^{\dagger}\left(\beta+\frac{1}{2}\right) P_{2,2}^{(9)} \underline{u} \wedge \underline{u}^{\dagger} \\
\underline{z}^{\dagger}\left(\beta+\frac{1}{2}\right) P_{2,2}^{(10)}|\underline{u}|^{2} \\
\underline{z}^{\dagger} P_{2,2}^{(11)} \underline{u} \wedge \underline{u}^{\dagger}
\end{array}\right),
\end{gathered}
$$

while the new contribution, again in comparison to the case $(1,1)$, comes from the action of $\underline{\partial}_{z}$ on the scalar 
factor $|\underline{z}|^{2}|\underline{u}|^{2}$. This gives

$$
\begin{gathered}
S(1,1) \underline{\dagger}^{\dagger} P_{2,2}^{(6)}|\underline{u}|^{2} \\
+S(0,1)\left(\frac{1}{2}|\underline{z}|^{2}|\underline{u}|^{2} P_{2,2}^{(7)} \underline{u}^{\dagger}-|\underline{u}|^{2} P_{2,2}^{(7)} \underline{z} \wedge \underline{z}^{\dagger} \underline{u}^{\dagger}\right) \\
+|\underline{z}|^{2}|\underline{u}|^{2}\left(\frac{1}{2} \underline{z}^{\dagger} P_{2,2}^{(9)} \underline{u} \wedge \underline{u}^{\dagger}+\frac{1}{2} \underline{z}^{\dagger} P_{2,2}^{(10)}|\underline{u}|^{2}\right. \\
\left.+\underline{z}^{\dagger} P_{2,2}^{(11)} \underline{u} \wedge \underline{u}^{\dagger}\right) .
\end{gathered}
$$

This means that all terms, except for the first and the third one, can be added to one of the terms coming from the obvious contribution. The first term must be added to terms coming from the zeroth row, while the third term can be rewritten as

$$
\begin{aligned}
& S(0,1)|\underline{u}|^{2} P_{2,2}^{(7)}\left(\underline{z}^{\dagger} \wedge \underline{z} \underline{u}^{\dagger}\right. \\
&= S(1,1) P_{2,2}^{(7)} \underline{z}^{\dagger}|\underline{u}|^{2} \\
&-S(0,1) \underline{u} \underline{u}^{\dagger}|\underline{u}|^{2} \underline{z} \wedge \underline{z}^{\dagger}\left(P_{2,2}^{(7)}+\left[P_{2,2}^{(7)}\right]\right) .
\end{aligned}
$$

In the end, this means that the contributions of $\underline{\partial}_{z}$ give rise to six equations that can be considered as the analogues of the equations found for the case $(1,1)$, together with a new equation coming from the terms in $\underline{u}^{\dagger}|\underline{u}|^{2} \underline{z} \wedge \underline{z}^{\dagger}$.

Since the equations coming from $\underline{\partial}_{z}^{\dagger}$ are very similar, we continue by giving the system of equations determining the spin-Euler polynomials of type $(2,2)$. The equations coming from the first row are given by

$$
\begin{aligned}
1+(1+\beta) P_{2,2}^{(1)} & =0, \\
\left(\frac{3}{2}+\beta\right) P_{2,2}^{(3)}+P_{2,2}^{(5)}+P_{2,2}^{(2)}+\left[P_{2,2}^{(2)}\right]^{\dagger} & =0,
\end{aligned}
$$

and

$$
\begin{gathered}
\left(\frac{1}{2}+\beta\right) P_{2,2}^{(4)}+P_{2,2}^{(6)}-\frac{1}{2} P_{2,2}^{(2)}-\frac{1}{2}\left[P_{2,2}^{(2)}\right]^{\dagger} \\
=\frac{1}{2} P_{2,2}^{(3)}-\left(P_{2,2}^{(7)}+\left[P_{2,2}^{(7)}\right]\right) .
\end{gathered}
$$

The third and sixth equations seem a bit strange, but the right-hand side is precisely taken care of by the equation coming from the exceptional terms $\underline{u}^{\dagger}\left(\underline{z} \wedge \underline{z}^{\dagger}\right)$ and $\underline{u}\left(\underline{z} \wedge \underline{z}^{\dagger}\right):$

$$
\frac{1}{2} P_{2,2}^{(3)}+P_{2,2}^{(4)}-\left(P_{2,2}^{(7)}+\left[P_{2,2}^{(7)}\right]\right)=0 .
$$

Indeed, this turns the third equation into

$$
\left(\frac{3}{2}+\beta\right) P_{2,2}^{(4)}+P_{2,2}^{(6)}-\frac{1}{2} P_{2,2}^{(2)}-\frac{1}{2}\left[P_{2,2}^{(2)}\right]^{\dagger}=0 .
$$

The equations from the second row are given by

$$
\begin{aligned}
\frac{1}{2} P_{2,2}^{(5)}+P_{2,2}^{(6)}+\left(\beta+\frac{3}{2}\right)\left(P_{2,2}^{(7)}+\left[P_{2,2}^{(7)}\right]\right) & =0, \\
(1+\beta) P_{2,2}^{(9)}+2 P_{2,2}^{(11)}+\left(P_{2,2}^{(8)}+\left[P_{2,2}^{(8)}\right]^{\dagger}\right) & =0, \\
(1+\beta) P_{2,2}^{(10)}+2 P_{2,2}^{(12)}-\frac{1}{2}\left(P_{2,2}^{(8)}+\left[P_{2,2}^{(8)}\right]^{\dagger}\right) & =0 .
\end{aligned}
$$

In a completely similar way, the equations coming from the action of $\underline{\partial}_{z}^{\dagger}$ can be derived:

$$
\begin{aligned}
1+(1+n-\beta) P_{2,2}^{(2)} & =0, \\
\left(\beta-n-\frac{3}{2}\right) P_{2,2}^{(3)}+P_{2,2}^{(5)}-P_{2,2}^{(1)}-\left[P_{2,2}^{(1)}\right] & =0, \\
\left(\beta-n-\frac{3}{2}\right) P_{2,2}^{(4)}+P_{2,2}^{(6)}-\frac{1}{2} P_{2,2}^{(1)}-\frac{1}{2}\left[P_{2,2}^{(1)}\right] & =0, \\
-\frac{1}{2} P_{2,2}^{(5)}+P_{2,2}^{(6)}+\left(n-\frac{1}{2}-\beta\right)\left(P_{2,2}^{(8)}+\left[P_{2,2}^{(8)}\right]^{\dagger}\right) & =0, \\
(\beta-n-1) P_{2,2}^{(9)}+2 P_{2,2}^{(11)}-\left(P_{2,2}^{(7)}+\left[P_{2,2}^{(7)}\right]\right) & =0, \\
(\beta-n-1) P_{2,2}^{(10)}+2 P_{2,2}^{(12)}-\left(\frac{1}{2} P_{2,2}^{(7)}+\frac{1}{2}\left[P_{2,2}^{(7)}\right]\right) & =0,
\end{aligned}
$$

where we have already used the exceptional equation

$$
P_{2,2}^{(4)}-\frac{1}{2} P_{2,2}^{(3)}+\left(P_{2,2}^{(8)}+\left[P_{2,2}^{(8)}\right]^{\dagger}\right)=0 .
$$

We immediately get that $P_{2,2}^{(1)}=P_{2}(\beta)$ and $P_{2,2}^{(2)}=$ $P_{2}(n-\beta)$. The polynomials $P_{2,2}^{(3)}, \ldots, P_{2,2}^{(6)}$ are easily obtained and have the same structure as the ones found in the $(1,1)$ case.

$$
\begin{aligned}
P_{2,2}^{(3)} & =-\frac{P_{3}(\beta)+P_{3}(n-\beta)}{(n+3)}, \\
P_{2,2}^{(4)} & =\frac{P_{3}(n-\beta)-P_{3}(\beta)}{2(n+3)}, \\
P_{2,2}^{(5)} & =-P_{3}(n-\beta)+\left(\beta+\frac{3}{2}\right) \frac{P_{3}(\beta)+P_{3}(n-\beta)}{(n+3)}, \\
P_{2,2}^{(6)} & =\frac{1}{2}\left(P_{3}(n-\beta)+\left(\beta+\frac{3}{2}\right) \frac{P_{3}(\beta)-P_{3}(n-\beta)}{(n+3)}\right) .
\end{aligned}
$$

From the exceptional equations, it follows that $P_{2,2}^{(7)}+$ $\left[P_{2,2}^{(7)}\right]=\frac{1}{n+3} P_{3}(\beta)$, leading to

$$
P_{2,2}^{(7)}(\beta)=-\frac{P_{2}(\beta)}{n+3} .
$$

In a completely similar way, it is immediately found that

$$
P_{2,2}^{(8)}(\beta)=-\frac{P_{2}(n-\beta)}{n+3} .
$$


Manipulating the other equations, we then obtain

$$
\begin{aligned}
& P_{2,2}^{(9)}= \frac{P_{2}(\beta)+P_{2}(n-\beta)}{(n+3)(n+2)}=-\frac{P_{2,2}^{(3)}}{n+2}, \\
& P_{2,2}^{(10)}= \frac{P_{2}(\beta)-P_{2}(n-\beta)}{2(n+3)(n+2)}=-\frac{P_{2,2}^{(4)}}{n+2}, \\
& P_{2,2}^{(11)}= \frac{1}{2(n+3)}\left(P_{2}(n-\beta)\right. \\
&\left.\quad-(1+\beta) \frac{P_{2}(\beta)+P_{2}(n-\beta)}{(n+2)}\right), \\
& P_{2,2}^{(12)}=-\frac{1}{4(n+3)}\left(P_{2}(n-\beta)\right. \\
&\left.\quad+(1+\beta) \frac{P_{2}(\beta)-P_{2}(n-\beta)}{(n+2)}\right) .
\end{aligned}
$$

\subsection{The General Case}

We first introduce notation for all spin-Euler polynomials occurring in the expression for $Z_{k, k}\left(\underline{z}, \underline{z}^{\dagger} ; \underline{u}, \underline{u^{\dagger}}\right)$. We will label these polynomials $P_{k, k}^{(j)}$, where $j$ goes from 1 to $6 k$, according to the scheme below. Let the Hermitian polynomial $Z_{k, k}\left(\underline{z}, \underline{z}^{\dagger} ; \underline{u}, \underline{u}^{\dagger}\right)$ be given by

$$
\begin{aligned}
& \sum_{j=0}^{k}|\underline{z}|^{2 j}|\underline{u}|^{2 j} \\
& \quad \times\left(\begin{array}{c}
\left(\underline{z} P_{k, k}^{(6 j+1)} S(k-j-1, k-j) \underline{u}^{\dagger}+\underline{z}^{\dagger} P_{k, k}^{(6 j+2)} S(k-j, k-j-1) \underline{u}\right) \\
S(k-j-1, k-j-1) \underline{z} \wedge \underline{z}^{\dagger} P_{k, k}^{(6 j+3)} \underline{u}^{\wedge} \underline{u}^{\dagger} \\
P_{k, k}^{(6 j)} S(k-j-1, k-j-1)\left(\underline{z} \wedge \underline{z}^{\dagger} P_{k, k}^{(6 j+4)}|\underline{u}|^{2}+|\underline{z}|^{2} P_{k, k}^{(6 j+5)} \underline{u} \wedge \underline{u}^{\dagger}\right)
\end{array}\right) .
\end{aligned}
$$

A few words of explanation might be useful here:

- $Z_{k, k}\left(\underline{z}, \underline{z}^{\dagger} ; \underline{u}, \underline{u}^{\dagger}\right)$ is defined in terms of $6 k$ polynomials. In order to determine these, we expect $6 k$ equations (we will find more equations, but they can be reduced to a system of $6 k$ equations). Inspired by the previous cases, we expect a rearrangement of all terms into $k$ times 6 equations $E_{j}^{(l)}$ (with $j \in\{0, \ldots, k-1\}$ and $l \in\{1, \ldots, 6\})$ :

$$
\begin{aligned}
\underline{u}^{\dagger} E_{j}^{(1)} S(k-j-1, k-j) & =0, \\
\underline{u} E_{j}^{(2)} S(k-j, k-j-1) & =0, \\
\underline{z}^{\dagger} E_{j}^{(3)} \underline{u} \wedge \underline{u}^{\dagger} S(k-j-1, k-j-1) & =0, \\
\underline{z} E_{j}^{(4)} \underline{u} \wedge \underline{u}^{\dagger} S(k-j-1, k-j-1) & =0, \\
\underline{z}^{\dagger} E_{j}^{(5)}|\underline{u}|^{2} S(k-j-1, k-j-1) & =0, \\
\underline{z} E_{j}^{(6)}|\underline{u}|^{2} S(k-j-1, k-j-1) & =0 .
\end{aligned}
$$

Each $E_{j}^{(l)}$ represents an equation containing spinEuler polynomials $P_{k, k}^{(i)}$ and polynomials $\left[P_{k, k}^{(i)}\right]$ or
$\left[P_{k, k}^{(i)}\right]^{+}$coming from a commutator with $\underline{u}$ or $\underline{u}^{\dagger}$. In view of the equations that were found for $(k, k)=$ $(2,2)$, we also expect additional exceptional equations, allowing us to simplify the equations $E_{j}^{(l)}$. These will be labeled by $\mathcal{E}_{j}$ and $\mathcal{E}_{j}^{\dagger}$, where $j$ refers to the fact that these equations come from

$$
\begin{aligned}
& \underline{u}^{\dagger} \underline{z} \wedge \underline{z}^{\dagger}|\underline{u}|^{2} S(k-j-2, k-j-1) \mathcal{E}_{j} \\
& \underline{u} \underline{z} \wedge \underline{z}^{\dagger}|\underline{u}|^{2} S(k-j-1, k-j-2) \mathcal{E}_{j}^{\dagger} .
\end{aligned}
$$

- The polynomials $P_{k, k}^{(6 j+1)}(\beta), \ldots, P_{k, k}^{(6 j+5)}(\beta)$ (with $j=0, \ldots, k-1)$ always belong together, in the sense that these have a factor $\left(|\underline{z}|^{2}|\underline{u}|^{2}\right)^{j}$ in common. We call these the polynomials of the $j$ th row. Note that the $k$ th row has only one term $|\underline{z}|^{2 k}|\underline{u}|^{2 k} P_{k, k}^{(6 k)}$, due to the fact that we put $S(a, b)=0$ as soon as one of these indices becomes zero.

- Given an index $l=6 j+r \in\{1,2, \ldots, 6 k\}$, it is always possible to determine to which term the corresponding polynomial $P_{k, k}^{(l)}$ belongs by means of the following procedure:

$$
\begin{aligned}
r & =0 \longrightarrow S(k-j, k-j), \\
r & =1 \longrightarrow \underline{z} \underline{u}^{\dagger} S(k-j-1, k-j), \\
r & =2 \longrightarrow \underline{z}^{\dagger} \underline{u} S(k-j, k-j-1), \\
r & =3 \longrightarrow \underline{z} \wedge \underline{z}^{\dagger} \underline{u} \wedge \underline{u}^{\dagger} S(k-j-1, k-j-1), \\
r & =4 \longrightarrow \underline{z} \wedge \underline{z}^{\dagger}|\underline{u}|^{2} S(k-j-1, k-j-1), \\
r & =5 \longrightarrow|\underline{z}|^{2} \underline{u} \wedge \underline{u}^{\dagger} S(k-j-1, k-j-1) .
\end{aligned}
$$

To determine these polynomials, we consider the action of $\underline{\partial}_{z}$ and $\underline{\partial}_{z}^{\dagger}$ on $Z_{k, k}\left(\underline{z}, \underline{z}^{\dagger} ; \underline{u}, \underline{u}^{\dagger}\right)$. Let us start with row zero (we prefer to count the rows from zero, in view of the power $j$ of the scalar factor $|\underline{u}|^{2}|\underline{z}|^{2}$ ). In what follows, all terms coming from the action of the operator $\underline{\partial}_{z}$ are listed, and the equation to which it will contribute is given in the second column:

$$
\begin{gathered}
S(k-1, k) \underline{u}^{\dagger} \rightarrow E_{0}^{(1)}, \\
(\beta+k-1) P_{k, k}^{(1)} S(k-1, k) \underline{u}^{\dagger} \rightarrow E_{0}^{(1)}, \\
-\underline{z}^{\dagger}\left(P_{k, k}^{(2)}+\left[P_{k, k}^{(2)}\right]^{\dagger}\right) S(k-1, k-1) \underline{u}^{\dagger} \underline{u} \rightarrow E_{0}^{(3)} / E_{0}^{(5)}, \\
\underline{z}^{\dagger}\left(\beta+\frac{1}{2}\right) P_{k, k}^{(3)} S(k-1, k-1) \underline{u} \wedge \underline{u}^{\dagger} \rightarrow E_{0}^{(3)}, \\
\underline{u}^{\dagger} \underline{z} \wedge \underline{z}^{\dagger} P_{k, k}^{(3)} S(k-2, k-1) \underline{u} \wedge \underline{u}^{\dagger} \rightarrow ?,
\end{gathered}
$$




$$
\begin{aligned}
& \underline{z}^{\dagger}\left(\beta+\frac{1}{2}\right) P_{k, k}^{(4)} S(k-1, k-1)|\underline{u}|^{2} \rightarrow E_{0}^{(5)}, \\
& \underline{u}^{\dagger} \underline{z} \wedge \underline{z}^{\dagger} P_{k, k}^{(4)} S(k-2, k-1)|\underline{u}|^{2} \rightarrow ?, \\
& \underline{z}^{\dagger} P_{k, k}^{(5)} S(k-1, k-1) \underline{u} \wedge \underline{u}^{\dagger} \rightarrow E_{0}^{(3)}, \\
& \underline{u}^{\dagger}|\underline{z}|^{2} P_{k, k}^{(5)} S(k-2, k-1) \underline{u} \wedge \underline{u}^{\dagger} \rightarrow ? .
\end{aligned}
$$

There are three terms for which the corresponding equation is not a priori clear. The last one, given by

$$
\begin{aligned}
& \underline{u}^{\dagger}|\underline{z}|^{2} P_{k, k}^{(5)} S(k-2, k-1) \underline{u} \wedge \underline{u}^{\dagger} \\
& =\frac{1}{2}|\underline{z}|^{2}|\underline{u}|^{2} S(k-2, k-1) \underline{u}^{\dagger} P_{k, k}^{(5)},
\end{aligned}
$$

contributes to the equation $E_{1}^{(1)}$. In other words, the action of the operator $\underline{\partial}_{z}$ on the $j$ th row will always give a term that mixes up with the unknown polynomials of the $(j+1)$ th row (except of course for the last row). Using the fact that

$$
\begin{aligned}
\underline{u}^{\dagger}(\underline{z} & \left.\wedge \underline{z}^{\dagger}\right)\left(\underline{u} \wedge \underline{u}^{\dagger}\right) \\
& =\frac{1}{2} \underline{u}^{\dagger}|\underline{u}|^{2} \underline{z} \wedge \underline{z}^{\dagger}-S(1,0) \underline{z}^{\dagger}\left(\frac{1}{2}|\underline{u}|^{2}-\underline{u} \wedge \underline{u}^{\dagger}\right),
\end{aligned}
$$

it is clear that the remaining terms will contribute to equations $E_{0}^{(3)}$ and $E_{0}^{(5)}$, and to equation $\mathcal{E}_{0}$ :

$$
|\underline{u}|^{2} \underline{u^{\dagger}} \underline{z} \wedge \underline{z}^{\dagger} S(k-2, k-1)\left(\frac{1}{2} P_{k, k}^{(3)}+P_{k, k}^{(4)}\right) .
$$

Similar conclusions will of course hold for the terms coming from the action of the operator $\underline{\partial}_{z}^{\dagger}$.

Let us now consider the $j$ th row (with $j>0$ ), i.e., the scalar factor $|\underline{z}|^{2 j}|\underline{u}|^{2 j}$ multiplied by the (six) terms inside parentheses. We again focus on the action of $\underline{\partial}_{z}$. This operator acts on the scalar term $|\underline{u}|^{2 j}|\underline{z}|^{2 j}$ as

$$
\underline{\partial}_{z}\left(|\underline{z}|^{2}|\underline{u}|^{2}\right)^{j}=j \underline{z}^{\dagger}|\underline{z}|^{2 j-2}|\underline{u}|^{2 j}
$$

and on the terms in parentheses. When acting on the terms in parentheses, we get at most three kinds of terms: terms contributing to equations $E_{j}^{(l)}$, a term contributing to $E_{j+1}^{(1)}$, and two exceptional terms contributing to $\mathcal{E}_{j}$.

For $j=k-1$ and $j=k$, however, the situation is different, since there will be no exceptional terms there. That is why in the special case $(1,1)$ we didn't observe this peculiar behavior.

Since the term in (4-2) is still to be multiplied by the six terms in parentheses, we get the following contributions:

$$
\begin{aligned}
& j\left(|\underline{z}|^{2}|\underline{u}|^{2}\right)^{j-1} \underline{z}^{\dagger} P_{k, k}^{(6 j)} S(k-j, k-j)|\underline{u}|^{2}, \\
& j\left(|\underline{z}|^{2}|\underline{u}|^{2}\right)^{j-1}\left(\frac{1}{2}|\underline{z}|^{2}-\underline{z} \wedge \underline{z^{\dagger}}\right) P_{k, k}^{(6 j+1)} \\
& \quad \times S(k-j-1, k-j) \underline{u^{\dagger}}|\underline{u}|^{2}, \\
& j\left(|\underline{z}|^{2}|\underline{u}|^{2}\right)^{j} \underline{z}^{\dagger} P_{k, k}^{(6 j+3)} S(k-j-1, k-j-1) \underline{u} \wedge \underline{u}^{\dagger}, \\
& j\left(|\underline{z}|^{2}|\underline{u}|^{2}\right)^{j} \underline{z}^{\dagger} P_{k, k}^{(6 j+4)} S(k-j-1, k-j-1)|\underline{u}|^{2}, \\
& j\left(|\underline{z}|^{2}|\underline{u}|^{2}\right)^{j} \underline{z}^{\dagger} P_{k, k}^{(6 j+5)} S(k-j-1, k-j-1) \underline{u} \wedge \underline{u}^{\dagger} .
\end{aligned}
$$

The first term reintroduces $P_{k, k}^{(6 j)}$, into equation $E_{j-1}^{(5)}$. All the other terms, except the third one, contribute to the equations $E_{j}^{(l)}$. Using the fact that $\left(\underline{z} \wedge \underline{z}^{\dagger}\right) \underline{u}^{\dagger}=$ $S(1,0) \underline{z}^{\dagger}-\underline{u}^{\dagger} \underline{z} \wedge \underline{z}^{\dagger}$, we can see that the third term will eventually contribute to $E_{j-1}^{(5)}$ and $\mathcal{E}_{j-1}$.

Straightforward (albeit elaborate) calculations yield the following global form for all spin-Euler polynomials of type $(k, k)$, up to the first two equations:

$$
\begin{aligned}
& E_{0}^{(1)}: 1+(\beta+k-1) P_{k, k}^{(1)}=0, \\
& E_{0}^{(2)}: 1+(n-\beta+k-1) P_{k, k}^{(2)}=0, \\
& E_{i}^{(1)}: \frac{1}{2} P_{k, k}^{(6 i-1)}+P_{k, k}^{(6 i)} \\
& +\left(\beta+\frac{2 k-i}{2}\right)\left(P_{k, k}^{(6 i+1)}+\left[P_{k, k}^{(6 i+1)}\right]\right)=0, \\
& E_{i}^{(2)}:-\frac{1}{2} P_{k, k}^{(6 i-1)}+P_{k, k}^{(6 i)} \\
& +\left(n-\beta+\frac{2 k-i}{2}\right)\left(P_{k, k}^{(6 i+2)}+\left[P_{k, k}^{(6 i+2)}\right]^{\dagger}\right)=0, \\
& E_{i}^{(3)}:\left(\beta+\frac{2 k-i-1}{2}\right) P_{k, k}^{(6 i+3)}+(i+1) P_{k, k}^{(6 i+5)} \\
& +\left(P_{k, k}^{(6 i+2)}+\left[P_{k, k}^{(6 i+2)}\right]^{\dagger}\right)=0, \\
& E_{i}^{(4)}:\left(\beta-n-\frac{2 k-i-1}{2}\right) P_{k, k}^{(6 i+3)}+(i+1) P_{k, k}^{(6 i+5)} \\
& -\left(P_{k, k}^{(6 i+1)}+\left[P_{k, k}^{(6 i+1)}\right]\right)=0, \\
& E_{i}^{(5)}:\left(\beta+\frac{i+1}{2}\right) P_{k, k}^{(6 i+4)}+(i+1) P_{k, k}^{(6 i+6)} \\
& -\frac{1}{2}\left(P_{k, k}^{(6 i+2)}+\left[P_{k, k}^{(6 i+2)}\right]^{\dagger}\right) \\
& +(i+1)(k-i-1)\left(P_{k, k}^{(6 i+7)}+\left[P_{k, k}^{(6 i+7)}\right]\right) \\
& -\frac{k-i-1}{2} P_{k, k}^{(6 i+3)}=0, \\
& E_{i}^{(6)}:\left(\beta-n-\frac{i+1}{2}\right) P_{k, k}^{(6 i+4)}+(i+1) P_{k, k}^{(6 i+6)} \\
& -\frac{1}{2}\left(P_{k, k}^{(6 i+1)}+\left[P_{k, k}^{(6 i+1)}\right]\right) \\
& +(i+1)(k-i-1)\left(P_{k, k}^{(6 i+8)}+\left[P_{k, k}^{(6 i+8)}\right]^{\dagger}\right) \\
& -\frac{k-i-1}{2} P_{k, k}^{(6 i+3)}=0 \text {, }
\end{aligned}
$$




$$
\begin{gathered}
\mathcal{E}_{i}:-(i+1)\left(P_{k, k}^{(6 i+7)}+\left[P_{k, k}^{(6 i+7)}\right]\right)+P_{k, k}^{(6 i+4)}+\frac{1}{2} P_{k, k}^{(6 i+3)}=0, \\
\mathcal{E}_{i}^{+}:(i+1)\left(P_{k, k}^{(6 i+8)}+\left[P_{k, k}^{(6 i+8)}\right]^{\dagger}\right)+P_{k, k}^{(6 i+4)}-\frac{1}{2} P_{k, k}^{(6 i+3)}=0 .
\end{gathered}
$$

This system is then solved recursively:

$$
\begin{aligned}
& P_{k, k}^{(1)}(\beta)=P_{k}(\beta), \\
& P_{k, k}^{(2)}(\beta)=P^{k}(\beta)=P_{k}(n-\beta) .
\end{aligned}
$$

It is easily shown (by induction) that

$$
\begin{aligned}
P_{k, k}^{(6 i+1)}= & \frac{(-1)^{i} \Gamma(n+2 k-i)}{i ! \Gamma(n+2 k)} P_{k}(\beta), \\
P_{k, k}^{(6 i+2)}= & \frac{(-1)^{i} \Gamma(n+2 k-i)}{i ! \Gamma(n+2 k)} P_{k}(n-\beta), \\
P_{k, k}^{(6 i+3)}= & \frac{(-1)^{i+1} \Gamma(n+2 k-i-1)}{i ! \Gamma(n+2 k)}\left(P_{k+1}(\beta)+P_{k+1}(n-\beta)\right), \\
P_{k, k}^{(6 i+4)}= & \frac{(-1)^{i+1} \Gamma(n+2 k-i-1)}{2 i ! \Gamma(n+2 k)}\left(P_{k+1}(\beta)-P_{k+1}(n-\beta)\right), \\
P_{k, k}^{(6 i+5)}= & \frac{(-1)^{i} \Gamma(n+2 k-i)}{(i+1) ! \Gamma(n+2 k)}\left(-P_{k+1}(n-\beta)\right. \\
& \left.+\left(\beta+\frac{2 k-i-1}{2}\right) \frac{P_{k+1}(\beta)+P_{k+1}(n-\beta)}{n+2 k-i-1}\right), \\
P_{k, k}^{(6 i+6)}= & \frac{(-1)^{i} \Gamma(n+2 k-i)}{2(i+1) ! \Gamma(n+2 k)}\left(P_{k+1}(n-\beta)\right. \\
& \left.+\left(\beta+\frac{2 k-i-1}{2}\right) \frac{P_{k+1}(\beta)-P_{k+1}(n-\beta)}{n+2 k-i-1}\right) .
\end{aligned}
$$

\section{SPIN-EULER POLYNOMIALS OF TYPE $(k, l) \in \mathbb{N}_{0}^{2}(k \neq l)$}

The last cases are those in which the indices differ: $k \neq l$. Note that the case in which one of them is equal to zero has already been treated. We start from the following expression for $Z_{k, l}\left(\underline{z}, \underline{z}^{\dagger} ; \underline{u}, \underline{u}^{\dagger}\right.$ ) (where we first consider the case $k>l$ ):

$$
\begin{aligned}
& \sum_{j=0}^{l}\left(|\underline{z}|^{2}|\underline{u}|^{2}\right)^{j} \\
& \times\left(\begin{array}{l}
S(k-j, l-j) P_{k, l}^{(6 j)} S(1+k-l, 1) \\
\left(\underline{z} P_{k, l}^{(6 j+1)} S(k-j-1, l-j) \underline{u}^{\dagger}+\underline{z}^{\dagger} P_{k, l}^{(6 j+2)} S(k-j, l-j-1) \underline{u}\right) \\
S(k-j-1, l-j-1) \underline{z} \wedge \underline{z}^{\dagger} P_{k, l}^{(6 j+3)} \underline{u} \wedge \underline{u}^{\dagger} \\
S(k-j-1, l-j-1)\left(\underline{z} \wedge \underline{z}^{\dagger} P_{k, l}^{(6 j+4)}|\underline{u}|^{2}+|\underline{z}|^{2} P_{k, l}^{(6 j+5)} \underline{u} \wedge \underline{u}^{\dagger}\right)
\end{array}\right)
\end{aligned}
$$

Given an index $l=6 j+r \in\{1,2, \ldots, 6 l+1\}$, one can easily determine to which term this polynomial belongs in the expansion for $Z_{k, l}\left(\underline{z}, \underline{z}^{\dagger} ; \underline{u}, \underline{u}^{\dagger}\right)$, using the following procedure:

$$
\begin{aligned}
& r=0 \longrightarrow S(k-j, l-j), \\
& r=1 \longrightarrow \underline{z}^{\dagger} S(k-j-1, l-j),
\end{aligned}
$$

$$
\begin{aligned}
r & =2 \longrightarrow \underline{z}^{\dagger} \underline{u} S(k-j, l-j-1), \\
r & =3 \longrightarrow \underline{z} \wedge \underline{z}^{\dagger} \underline{u} \wedge \underline{u}^{\dagger} S(k-j-1, l-j-1), \\
r & =4 \longrightarrow \underline{z} \wedge \underline{z}^{\dagger}|\underline{u}|^{2} S(k-j-1, l-j-1), \\
r & =5 \longrightarrow|\underline{z}|^{2} \underline{u} \wedge \underline{u}^{\dagger} S(k-j-1, l-j-1) .
\end{aligned}
$$

To determine all these polynomials, we have to let $\underline{\partial}_{z}$ and $\underline{\partial}_{z}^{\dagger}$ act on $Z_{k, l}$. The calculations are very similar to those performed for the case $(k, k)$, up to some minor changes in the coefficients. Apart from the equations $E_{0}^{(1)}$ and $E_{0}^{(2)}$, given by

$$
\begin{aligned}
& E_{0}^{(1)}: 1+(\beta+k-1) P_{k, l}^{(1)}=0, \\
& E_{0}^{(2)}: 1+(n-\beta+l-1) P_{k, l}^{(2)}=0,
\end{aligned}
$$

and the fact that there is no equation $\mathcal{E}_{l-1}^{\dagger}$ (which is due to the fact that $k>l)$, all equations $E_{j}^{(r)}, \mathcal{E}_{j}$, and $\mathcal{E}_{j}^{\dagger}$ (with $j=0, \ldots, l-1$ and $r=1, \ldots, 6$ ) are given by

$$
\begin{aligned}
& E_{i}^{(1)}: \frac{1}{2} P_{k, l}^{(6 i-1)}+P_{k, l}^{(6 i)} \\
& +\left(\beta+\frac{2 k-i}{2}\right)\left(P_{k, l}^{(6 i+1)}+\left[P_{k, l}^{(6 i+1)}\right]\right)=0, \\
& E_{i}^{(2)}:-\frac{1}{2} P_{k, l}^{(6 i-1)}+P_{k, l}^{(6 i)} \\
& +\left(n-\beta+\frac{2 l-i}{2}\right)\left(P_{k, l}^{(6 i+2)}+\left[P_{k, l}^{(6 i+2)}\right]^{\dagger}\right)=0, \\
& E_{i}^{(3)}:\left(\beta+\frac{2 k-i-1}{2}\right) P_{k, l}^{(6 i+3)}+(i+1) P_{k, l}^{(6 i+5)} \\
& +\left(P_{k, l}^{(6 i+2)}+\left[P_{k, l}^{(6 i+2)}\right]^{\dagger}\right)=0, \\
& E_{i}^{(4)}:\left(\beta-n-\frac{2 l-i-1}{2}\right) P_{k, l}^{(6 i+3)}+(i+1) P_{k, l}^{(6 i+5)} \\
& -\left(P_{k, l}^{(6 i+1)}+\left[P_{k, l}^{(6 i+1)}\right]\right)=0, \\
& E_{i}^{(5)}:\left(\beta+\frac{i+1}{2}\right) P_{k, l}^{(6 i+4)}+(i+1) P_{k, l}^{(6 i+6)} \\
& -\frac{1}{2}\left(P_{k, l}^{(6 i+2)}+\left[P_{k, l}^{(6 i+2)}\right]^{\dagger}\right) \\
& +(i+1)(k-i-1)\left(P_{k, l}^{(6 i+7)}+\left[P_{k, l}^{(6 i+7)}\right]\right) \\
& -\frac{k-i-1}{2} P_{k, l}^{(6 i+3)}=0, \\
& E_{i}^{(6)}:\left(\beta-n-\frac{i+1}{2}\right) P_{k, l}^{(6 i+4)}+(i+1) P_{k, l}^{(6 i+6)} \\
& -\frac{1}{2}\left(P_{k, l}^{(6 i+1)}+\left[P_{k, l}^{(6 i+1)}\right]\right) \\
& +(i+1)(l-i-1)\left(P_{k, l}^{(6 i+8)}+\left[P_{k, l}^{(6 i+8)}\right]^{\dagger}\right) \\
& -\frac{l-i-1}{2} P_{k, l}^{(6 i+3)}=0 \text {, } \\
& \mathcal{E}_{i}:-(i+1)\left(P_{k, l}^{(6 i+7)}+\left[P_{k, l}^{(6 i+7)}\right]\right)+P_{k, l}^{(6 i+4)}+\frac{1}{2} P_{k, l}^{(6 i+3)}=0, \\
& \mathcal{E}_{i}^{+}:(i+1)\left(P_{k, l}^{(6 i+8)}+\left[P_{k, l}^{(6 i+8)}\right]^{\dagger}\right)+P_{k, l}^{(6 i+4)}-\frac{1}{2} P_{k, l}^{(6 i+3)}=0 .
\end{aligned}
$$


This system of equations can then easily be solved recursively, which gives rise to

$$
\begin{aligned}
P_{k, l}^{(6 i+1)}= & \frac{(-1)^{i} \Gamma(n+k+l-i)}{i ! \Gamma(n+k+l)} P_{k}(\beta), \\
P_{k, l}^{(6 i+2)}= & \frac{(-1)^{i} \Gamma(n+k+l-i)}{i ! \Gamma(n+k+l)} P_{l}(n-\beta), \\
P_{k, l}^{(6 i+3)}= & \frac{(-1)^{i+1} \Gamma(n+k+l-i-1)}{i ! \Gamma(n+k+l)}\left(P_{k+1}(\beta)+P_{l+1}(n-\beta)\right), \\
P_{k, l}^{(6 i+4)}= & \frac{(-1)^{i+1} \Gamma(n+k+l-i-1)}{2 i ! \Gamma(n+k+l)}\left(P_{k+1}(\beta)-P_{l+1}(n-\beta)\right), \\
P_{k, l}^{(6 i+5)}= & \frac{(-1)^{i} \Gamma(n+k+l-i)}{(i+1) ! \Gamma(n+k+l)}\left(-P_{l+1}(n-\beta)\right. \\
& \left.+\left(\beta+\frac{2 k-i-1}{2}\right) \frac{P_{k+1}(\beta)+P_{l+1}(n-\beta)}{n+k+l-i-1}\right), \\
P_{k, l}^{(6 i+6)}= & \frac{(-1)^{i} \Gamma(n+k+l-i)}{2(i+1) ! \Gamma(n+k+l)}\left(P_{l+1}(n-\beta)\right. \\
& \left.+\left(\beta+\frac{2 k-i-1}{2}\right) \frac{P_{k+1}(\beta)-P_{l+1}(n-\beta)}{n+k+l-i-1}\right) .
\end{aligned}
$$

For the case $k<l$, we of course get exactly the same polynomials.

We may then conclude this section with the following theorem:

Theorem 5.1. The building blocks for the Hermitian Fischer decomposition of the reproducing kernel $\mathcal{R}_{k, l}\left(\underline{z}, \underline{z}^{\dagger} ; \underline{u}, \underline{u}^{\dagger}\right)$ can all be expressed in terms of the spinEuler polynomials $P_{k}(\beta)$ and $P_{k}(n-\beta)$. For the explicit formulas, we refer to the previous sections.

\section{ACKNOWLEDGMENTS}

The author would like to thank Professor F. Sommen, of Ghent University, for his valuable suggestions and encouragement.

The author was supported as a postdoctoral fellow by the F. W. O.-Vlaanderen (Belgium).

\section{REFERENCES}

[Brackx et al. 82] F. Brackx, R. Delanghe, and F. Sommen. Clifford Analysis, Research Notes in Math. 76. London: Pitman, 1982.

[Brackx et al. 05] F. Brackx, H. De Schepper, and F. Sommen. "The Hermitian Clifford Analysis Toolbox." To appear in Proceedings of ICCAY (Toulouse, 2005).

[Delanghe et al. 92] R. Delanghe, F. Sommen, and V. Souček. Clifford Algebras and Spinor-Valued Functions. Dordrecht: Kluwer Academic Publishers, 1992.

[Gilbert and Murray 91] J. Gilbert and M. A. M. Murray. Clifford Algebras and Dirac Operators in Harmonic Analysis. Cambridge: Cambridge University Press, 1991.

[Graham et al. 94] R. L. Graham, D. E. Knuth, and O. Patashnik. Concrete Mathematics: A Foundation for Computer Science. Reading, MA: Addison-Wesley, 1994.

[Rocha-Chavez et al. 01] R. Rocha-Chavez, M. Shapiro, and F. Sommen. Integral Theorems for Functions and Differential Forms in $\mathbb{C}^{m}$, Research Notes in Math. 428. Boca Raton: Chapman and Hall/CRC Press, 2001.

[Sabadini and Sommen 02] I. Sabadini and F. Sommen. "Hermitian Clifford Analysis and Resolutions." $M M A S$ 25:16-18 (2002), 1395-1414.

D. Eelbode, Ghent University, Department of Mathematical Analysis, Clifford Research Group, Galglaan 2, 9000 Ghent, Belgium (deef@cage.UGent.be)

Received October 12, 2005; accepted in revised form May 30, 2006. 